\title{
Incidence and risk of hepatic toxicities with PD-I inhibitors in cancer patients: a meta-analysis
}

This article was published in the following Dove Press journal:

Drug Design, Development and Therapy

28 September 2016

Number of times this article has been viewed

\author{
Xi Zhang' \\ Yuge Ran' \\ Kunjie Wang ${ }^{2}$ \\ Yuanxue Zhu \\ Jinghua $\mathrm{Li}^{3}$ \\ 'Department of Radiation Oncology, \\ ${ }^{2}$ Department of Medical Oncology, \\ ${ }^{3}$ Department of Hepatobiliary Surgery, \\ Affiliated Hospital of Hebei University, \\ Baoding, People's Republic of China
}

Correspondence: Jinghua Li

Department of Hepatobiliary Surgery,

Affiliated Hospital of Hebei University,

21 2 East Yuhua Road, Baoding, 07/000

Hebei, People's Republic of China

Tel $+86312598 \quad 1810$

$\mathrm{Fax}+86312598 \quad 1152$

Email lijinghuamd@।63.com
Purpose: Anti-programmed cell death receptor-1 (PD-1) antibodies have demonstrated antitumor activity in many cancer entities. Hepatic adverse events (AEs) are one of its major side effects, but the overall risks have not been systematically evaluated. Thus, we conducted this meta-analysis to investigate the overall incidence and risk of developing hepatic AEs in cancer patients treated with PD-1 inhibitors.

Methods: PubMed, Embase, and oncology conference proceedings were searched for relevant studies. Eligible studies were randomized controlled trials of cancer patients treated with PD-1 inhibitors with adequate data on hepatic AEs.

Results: A total of nine randomized controlled trials with a variety of solid tumors were eligible for the meta-analysis. The use of PD-1 inhibitors significantly increased the risk of developing all-grade hepatic AEs but not for high-grade hepatic AEs in comparison with chemotherapy or everolimus control. Additionally, the risk of all-grade and high-grade hepatic AEs with a nivolumab/ipilimumab combination was substantially higher than ipilimumab. No significant differences in the risk of all-grade and high-grade hepatic AEs were found between PD-1 inhibitors monotherapy and ipilimumab.

Conclusion: While the use of PD-1 inhibitors is associated with an increased risk of developing hepatic AEs in cancer patients, this is primarily for lower grade events.

Keywords: cancer, hepatic toxicities, PD-1 inhibitors, meta-analysis

\section{Introduction}

Program death 1 (PD-1) and its ligand (PD-L1) have been identified as potential therapeutic targets for solid and hematologic malignancies in recent years. PD-1 is an inhibitory receptor, which prevents overactivation of T-cells and thus prevents autoimmunity by promoting tolerance to self-antigens and to limit damage to normal tissues. PD-1 and its ligands (PD-L1 and PD-L2) have been noted as critical mediators of immunosuppression within the tumor microenvironment. ${ }^{1,2}$ Tumor cells can suppress the activity of T-cells in tissues and the tumor microenvironment by binding PD-L1 to PD-1 on the T-cells to evade immune destruction. ${ }^{3}$ Recently, several antibodies that inhibit the interaction between PD-L1 and PD-1 have demonstrated durable efficacy in a number of solid and hematologic malignancies. ${ }^{47}$ Nivolumab is a fully human monoclonal immunoglobulin G4 anti-PD-1 antibody that has been approved for the treatment of unresectable or metastatic melanoma, advanced nonsmall-cell lung cancer, and advanced renal cell carcinoma. ${ }^{711}$ Pembrolizumab is another human PD-1-blocking antibody indicated for the treatment of patients with unresectable or metastatic melanoma. ${ }^{12}$ Moreover, it is being extensively evaluated as a single agent and as a combination with other therapeutic agents in many advanced refractory solid tumors. ${ }^{13}$ Other antibodies targeting the PD-1/PD-L1 checkpoint 
in the phase of development include atezolizumab and durvalumab, which have also shown activity against many solid malignancies. ${ }^{14-16}$ However, the very specific mechanism of action of those new medicines brings about some specific immunological adverse event (AE) profiles, which is different from toxicities known for cytotoxic drugs or kinase inhibitors. ${ }^{17}$ For instance, many clinical trials have shown that these side effects affect skin, lung, gastrointestinal tract, liver, endocrine system, and other organ systems. ${ }^{9-11}$ Hepatic AEs mainly manifest as asymptomatic increase of aspartate aminotransferase (AST) and alanine aminotransferase (ALT) levels. ${ }^{18}$ The contribution of anti-PD-1 therapy to the development of hepatic AEs is difficult to assess as individual randomized controlled trials (RCTs) may be not powered to detect a significant relationship. Although a meta-analysis has shown that the use of immune checkpoint inhibitors has a causal relationship to an increased risk of high-grade (grade 3 or 4) elevated ALT and AST, ${ }^{19}$ most RCTs included in the analysis evaluated anti-CTLA-4 monoclonal antibodies and only three RCTs evaluated anti-PD-1 antibody (nivolumab), thus the contributions of anti-PD-1 therapy to hepatic AEs are still unclear. As a result, we conducted a meta-analysis of all published RCTs to determine the incidence and risk of hepatic AEs associated with the use of PD-1 inhibitors in cancer patients.

\section{Methods}

\section{Data sources}

This meta-analysis was performed according to the PRISMA (Preferred Reporting Items for Systemic Reviews and Meta-Analyses) recommendations. ${ }^{20}$ A literature review of PubMed (data from January 2000 to May 2016) and Embase (data from January 2000 to May 2016) was conducted to identify relevant clinical trials. The search was conducted using keywords "nivolumab", "pembrolizumab", "cancer", and "trials". The search was limited to RCTs published in English. Additionally, we searched abstracts presented at the annual meetings of the American Society of Clinical Oncology and the European Society of Medical Oncology annual meetings. Moreover, the references cited by the included studies were also used to complete the search. Each publication was reviewed, and in cases of multiple publications of the same clinical trial were encountered, only the most complete, recent, and updated report of clinical trials was included in the meta-analysis.

\section{Study selection}

The primary objective of this analysis was to determine the overall incidence and relative risk (RR) of hepatic AEs associated with PD-1 inhibitors. Thus, Phase I and nonrandomized Phase II trials were excluded from the analysis due to lack of sufficient controls. The relevant clinical trials were selected carefully based on the following criteria: 1) prospective RCTs evaluating patients with solid tumors, 2) patients were allocated to treatment with PD-1 inhibitors (nivolumab $3 \mathrm{mg} / \mathrm{kg}$, pembrolizumab $2 \mathrm{mg} / \mathrm{kg}$ or $10 \mathrm{mg} / \mathrm{kg}$ ) or control (chemotherapy or everolimus or ipilimumab), and 3) available data regarding events or event rate and sample size of all-grade and high-grade hepatic AEs.

\section{Data extraction and clinical end point}

The data abstraction was conducted by two authors independently, and discrepancies were resolved by consensus. For each study, the following details were extracted: the name of first author, year of publication, trial phase, cancer type, treatment arms and control arms, and patients included for analysis. The following outcomes were considered as hepatic AEs: elevated ALT and elevated AST. AEs of all grade and high grade were defined according to the National Cancer Institute's common toxicity criteria (Version 4.0; http://ctep. cancer.gov) (Table 1). Study quality of RCTs was assessed

Table I Baseline characteristics of six trials comparing PD-I inhibitors to chemotherapy or everolimus

\begin{tabular}{|c|c|c|c|c|c|c|c|c|}
\hline Study & Phase & $\begin{array}{l}\text { Underlying } \\
\text { malignancy }\end{array}$ & Treatment arm & Control arm & $\begin{array}{l}\text { Treatment } \\
\text { arm }\end{array}$ & $\begin{array}{l}\text { Control } \\
\text { arm }\end{array}$ & $\begin{array}{l}\text { CTC } \\
\text { version }\end{array}$ & $\begin{array}{l}\text { Jadad } \\
\text { score }\end{array}$ \\
\hline Borghaei et al ${ }^{9}$ & III & NSCLC & Nivolumab 3 mg/kg every 2 weeks & Docetaxel & 287 & 268 & 4.0 & 3 \\
\hline Brahmer et al $^{10}$ & III & NSCLC & Nivolumab $3 \mathrm{mg} / \mathrm{kg}$ every 2 weeks & Docetaxel & $|3|$ & 129 & 4.0 & 3 \\
\hline Robert et $\mathrm{al}^{25}$ & III & Melanoma & Nivolumab 3 mg/kg every 2 weeks & Dacarbazine & 206 & 205 & 4.0 & 5 \\
\hline Weber et $\mathrm{al}^{7}$ & III & Melanoma & Nivolumab 3 mg/kg every 2 weeks & $\begin{array}{l}\text { Investigator's choice } \\
\text { of chemotherapy }\end{array}$ & 268 & 102 & 4.0 & 3 \\
\hline Motzer et al'" & III & $\mathrm{RCC}$ & Nivolumab 3 mg/kg every 2 weeks & Everolimus & 406 & 397 & 4.0 & 3 \\
\hline Herbst et $\mathrm{al}^{5}$ & II/III & NSCLC & $\begin{array}{l}\text { Pembrolizumab } 2 \mathrm{mg} / \mathrm{kg} \text { every } 3 \text { weeks } \\
\text { Pembrolizumab } 10 \mathrm{mg} / \mathrm{kg} \text { every } 3 \text { weeks }\end{array}$ & $\begin{array}{l}\text { Docetaxel } \\
\text { Docetaxel }\end{array}$ & $\begin{array}{l}339 \\
343\end{array}$ & 309 & 4.0 & 3 \\
\hline
\end{tabular}

Abbreviations: PD-I, program death I; CTC, common toxicity criteria; NSCLC, non-small-cell lung cancer; RCC, renal cell carcinoma. 
according to the Jadad scale including randomization, blinding, and withdrawals. ${ }^{21}$

\section{Statistical analysis}

The number of hepatic AEs and the total number of patients receiving PD-1 inhibitors were extracted from the safety profiles of all selected studies, and then the proportion of patients with hepatic AEs and 95\% confidence interval (CI) were calculated for each study. Studies that had a comparative arm were used to calculate the RR of hepatic AEs. For the metaanalysis, both the fixed-effect model and the random-effect model were considered. ${ }^{22,23}$ The $\chi^{2}$-based $Q$ statistic was first applied to estimate between-study heterogeneity, and inconsistency was quantified with the $I^{2}$ statistic, which estimates the percentage of total variation across studies because of heterogeneity rather than chance. ${ }^{24}$ Heterogeneity was considered statistically significant when $P<0.1$. If heterogeneity existed, data were analyzed using a random-effect model. Otherwise, a fixed-effect model was used. Moreover, the following subgroup analyses were conducted: 1) PD-1 inhibitors monotherapy (nivolumab or pembrolizumab) versus control (including chemotherapy or everolimus), 2) a nivolumab/ ipilimumab combination versus ipilimumab control, and 3) PD-1 inhibitors monotherapy (nivolumab or pembrolizumab) versus ipilimumab control. A two-tailed $P$-value of $<0.05$ was considered statistically significant. All statistical analyses were performed by using Version 2 of the Comprehensive Meta Analysis program (Biostat, Englewood, NJ, USA).

\section{Results \\ Search results}

On the basis of our search criteria, 251 potentially clinical studies evaluating nivolumab or pembrolizumab were identified. After the selection procedure, ten full-text articles were considered for further assessment; one trial without sufficient data on hepatic AEs was excluded. Finally, a total of nine $\mathrm{RCTs}^{5,7-12,25,26}$ were considered to be of adequate quality and relevance for the meta-analysis. Studies excluded from the analysis and the reasons for their exclusion are shown in Figure 1.

\section{Study characteristics}

In total, nine RCTs were available for the meta-analysis including eight Phase III trials and one randomized Phase II/III trial. Four studies evaluated nivolumab monotherapy versus chemotherapy controls, ${ }^{7,9,10,25}$ one study evaluated nivolumab monotherapy versus everolimus (data on high-grade hepatic AEs), ${ }^{11}$ one study evaluated

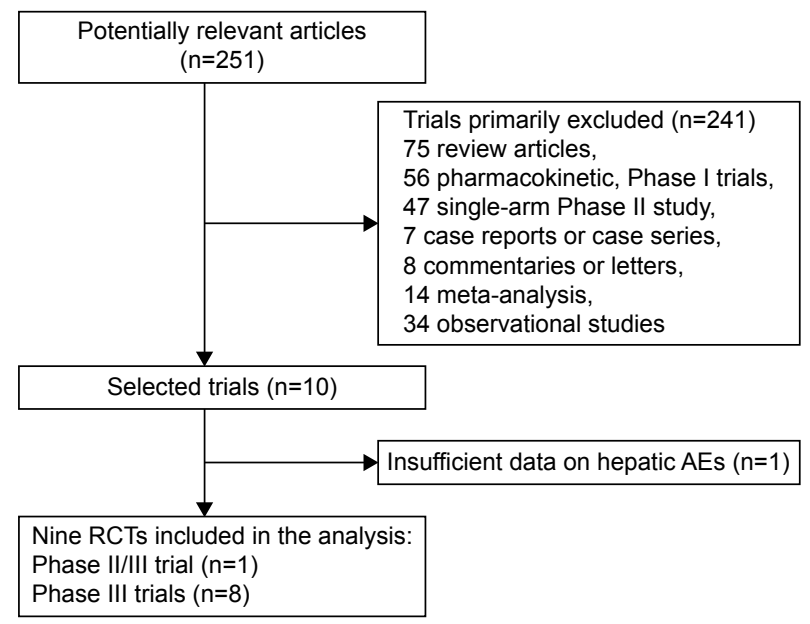

Figure I Flow chart of selection process for trials included in meta-analysis. Abbreviations: AEs, adverse events; RCTs, randomized controlled trials.

pembrolizumab monotherapy versus chemotherapy control, ${ }^{5}$ two studies evaluated nivolumab/ipilimumab combination versus ipilimumab, ${ }^{8,26}$ and two studies evaluated pembrolizumab monotherapy or nivolumab monotherapy versus ipilimumab control. ${ }^{12,26}$ Underlying malignancies included melanoma, ${ }^{7,8,12,25,26}$ renal cell carcinoma, ${ }^{11}$ and advanced non-small-cell lung cancer. ${ }^{5,9,10}$ All trials reported the hepatic AEs according to the National Cancer Institute's Common Terminology Criteria for Adverse Events version four criteria. Jadad scores were listed for each trial in Tables 1 and 2; the mean score was 3.7 (range, 3.0-5.0), indicating that the overall methodological quality of the included studies was good and fair.

\section{Incidence of all-grade and high-grade hepatic AEs}

For the incidence analysis, only arms receiving one of the anti PD-1 antibodies were included. A total of 2,442 patients from seven RCTs were included for the calculation of the incidence of all-grade hepatic AEs. The calculated summary incidence of all-grade hepatic AEs was 3.1\% (95\% CI: $2.5 \%-3.9 \%$ ) using the fixed-effect model (heterogeneity test: $\left.I^{2}=0 \%, P=0.67\right)$ for elevated ALT and 3.2\% (95\% CI: $2.5 \%-3.9 \%$ ) with the fixed-effect model (heterogeneity test: $I^{2}=0.82 \%, P=0.366$ ) for elevated AST (Figure 2). Data for high-grade hepatic AEs included a total of 2,848 patients from eight RCTs. The summary incidence of high-grade elevated ALT was $0.6 \%$ (95\% CI: $0.4 \%-1.1 \%$ ) according to the fixed-effect model (heterogeneity test: $I^{2}=0 \%, P=0.600$ ), and the calculated incidence of high-grade elevated AST was $0.5 \%$ (95\% CI: $0.3 \%-0.8 \%)$ using a fixed-effect model (heterogeneity test: $I^{2}=0 \%, P=0.879$ ) (Figure 2). 
Table 2 Direct comparison among different immune checkpoint inhibitors

\begin{tabular}{|c|c|c|c|c|c|c|c|c|}
\hline Study & Phase & $\begin{array}{l}\text { Underlying } \\
\text { malignancy }\end{array}$ & Treatment arm & $\begin{array}{l}\text { Control } \\
\text { arm }\end{array}$ & $\begin{array}{l}\text { Treatment } \\
\text { arm }\end{array}$ & $\begin{array}{l}\text { Control } \\
\text { arm }\end{array}$ & $\begin{array}{l}\text { CTC } \\
\text { version }\end{array}$ & $\begin{array}{l}\text { Jadad } \\
\text { score }\end{array}$ \\
\hline \multirow[t]{2}{*}{ Larkin et $\mathrm{al}^{26}$} & III & Melanoma & Nivolumab $3 \mathrm{mg} / \mathrm{kg}$ combined with placebo & Ipilimumab & 313 & 311 & 4.0 & 5 \\
\hline & & & Ipilimumab combined with nivolumab (I mg/kg) & Ipilimumab & 313 & & & \\
\hline Postow et a $\left.\right|^{8}$ & III & Melanoma & Ipilimumab combined with nivolumab (I mg/kg) & Ipilimumab & 94 & 46 & 4.0 & 5 \\
\hline \multirow[t]{2}{*}{ Robert et al ${ }^{12}$} & III & Melanoma & Pembrolizumab $10 \mathrm{mg} / \mathrm{kg}$ every 2 weeks & Ipilimumab & 278 & 256 & 4.0 & 3 \\
\hline & & & Pembrolizumab $10 \mathrm{mg} / \mathrm{kg}$ every 3 weeks & Ipilimumab & 277 & & & \\
\hline
\end{tabular}

Abbreviation: CTC, common toxicity criteria.

\section{RR of hepatic AEs in patients treated with PD-I inhibitors monotherapy versus chemotherapy or everolimus control}

The RR of hepatic AEs was calculated by comparing with those assigned to a control treatment in the same trial to determine the specific contribution of PD-1 inhibitors to the development of hepatic AEs. A total of 2,587 patients from five RCTs were included for calculating the RR of all-grade hepatic AEs, and the combined results demonstrated that the use of PD-1 inhibitors monotherapy was associated with a significantly increased risk of developing all-grade hepatic AEs with an RR of 2.09 (95\% CI: 1.1-3.95, $P=0.024)$ (heterogeneity test: $P=0 \%, P=0.889$ ) for elevated ALT and 2.02 (95\% CI: 1.01-4.03, $P=0.047$ ) (heterogeneity test:

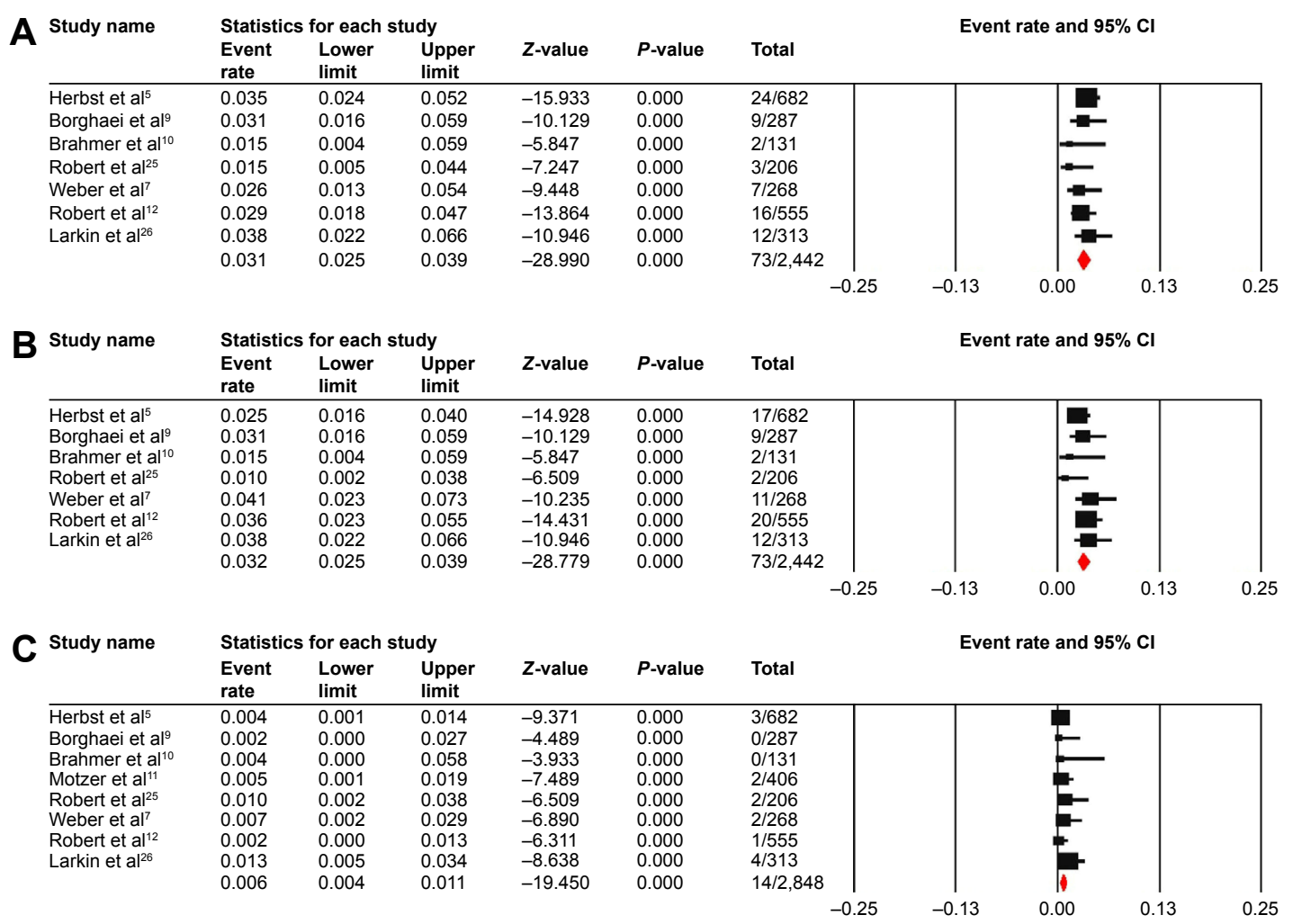

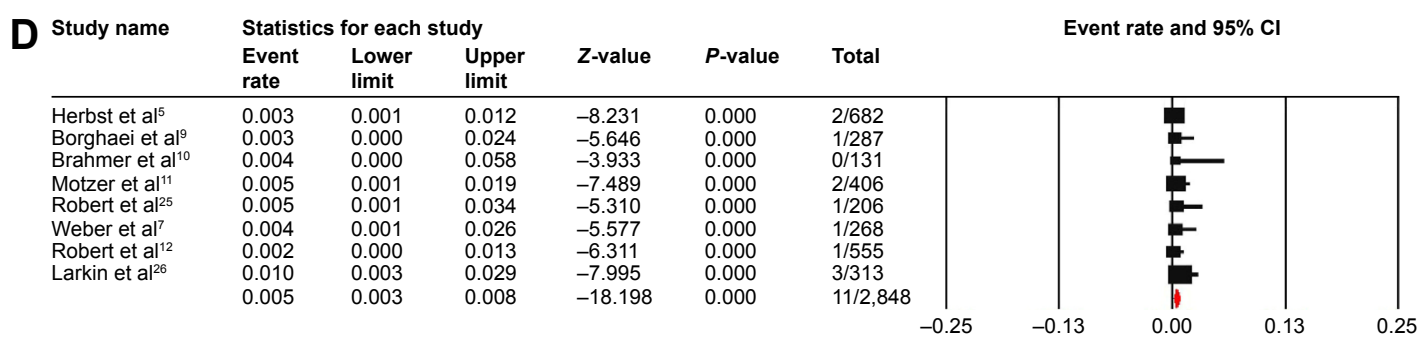

Figure 2 Forest plot for meta-analysis of incidence of all-grade elevated ALT (A) and AST (B) and high-grade elevated ALT (C) and AST (D). Abbreviations: ALT, alanine aminotransferase; AST, aspartate aminotransferase; $\mathrm{Cl}$, confidence interval. 
$I^{2}=0 \%, P=0.449$ ) for elevated AST (Figure 3). As for highgrade hepatic AEs, a total of 3,390 patients from six RCTs were included for analysis. The combined RR showed that the use of PD-1 inhibitors monotherapy did not significantly increase the risk of high-grade hepatic AEs among cancer patients with an RR of 1.47 (95\% CI: 0.45-4.86, $P=0.525$ ) (heterogeneity test: $P=0 \%, P=0.745$ ) for elevated ALT and 1.51 (95\% CI: $0.43-5.27, P=0.515$ ) (heterogeneity test: $I^{2}=0 \%, P=0.878$ ) for elevated AST (Figure 3 ).

\section{RR of hepatic AEs in patients treated with a nivolumab/ipilimumab combination versus ipilimumab control}

In order to determine whether a nivolumab/ipilimumab combination is associated with a higher risk of developing hepatic toxicities than ipilimumab monotherapy, we calculated the RR according to a nivolumab/ipilimumab combination versus ipilimumab control. The RR of allgrade elevated ALT and AST was 4.64 (95\% CI: 2.66-8.09, $P<0.001$ ) (heterogeneity test: $P=0 \%, P=0.877$ ) and 4.43 (95\% CI: 2.48-7.90, $P<0.001$ ) (heterogeneity test: $I^{2}=0 \%$, $P=0.878$ ), respectively (Figure 4). Moreover, there was a statistically significant increase in the risk of high-grade hepatic AEs with an RR of 5.55 (95\% CI: 2.27-13.57; $P<0.001$ ) (heterogeneity test: $P^{2}=0 \%, P=0.645$ ) for elevated ALT and 8.98 (95\% CI: 2.47-32.65; $P=0.001$ ) (heterogeneity test: $P^{2}=0 \%, P=0.882$ ) for elevated AST (Figure 4). Thus, compared with ipilimumab, a nivolumab/ipilimumab combination was associated with higher risk of developing all-grade and high-grade hepatic AEs.

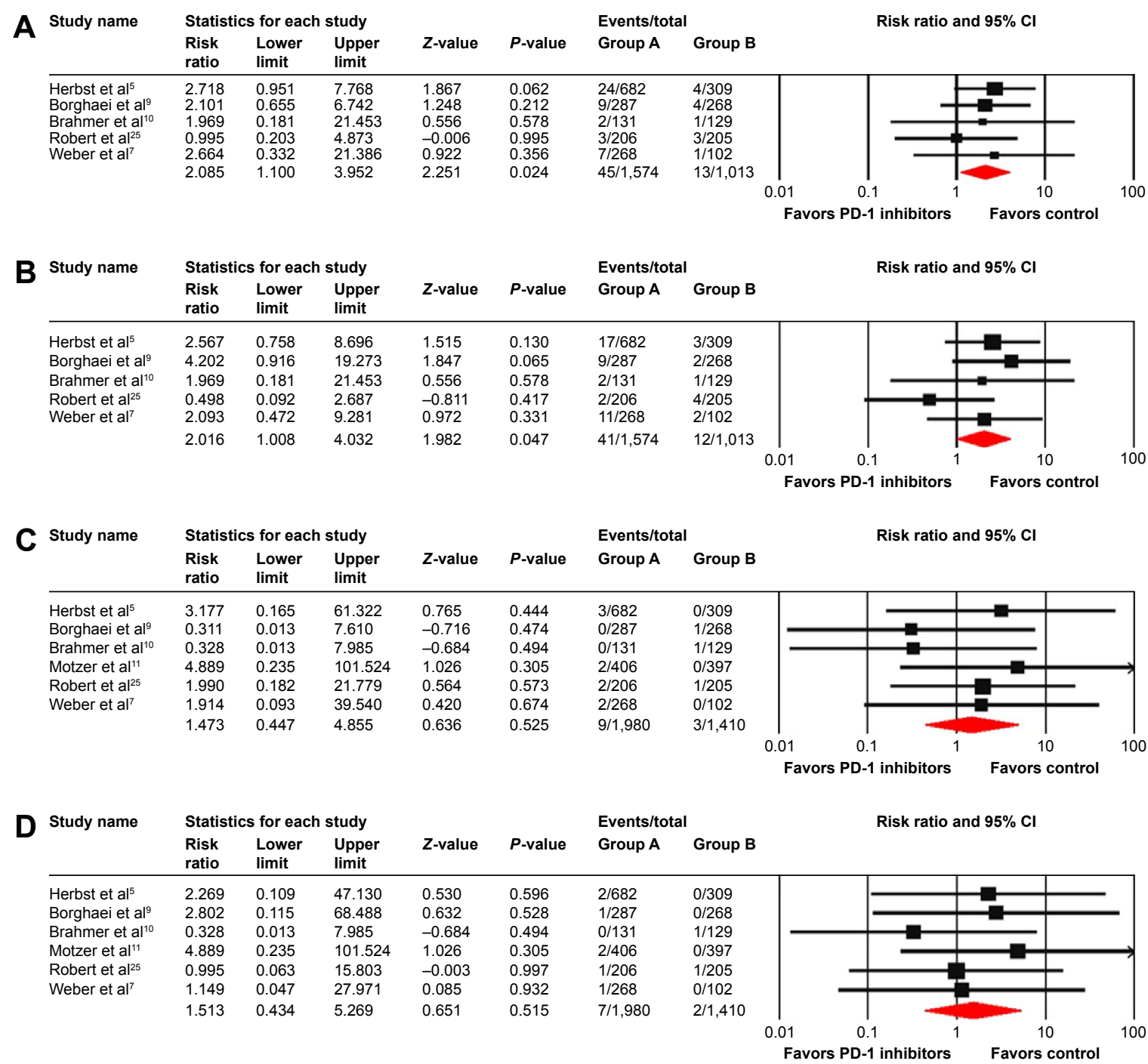

Figure 3 RR of all-grade elevated ALT (A) and AST (B) and high-grade elevated ALT (C) and AST (D) for cancer patients receiving PD-I inhibitors monotherapy compared with control.

Abbreviations: ALT, alanine aminotransferase; AST, aspartate aminotransferase; PD-I, program death I; Cl, confidence interval; RR, risk ratio. 


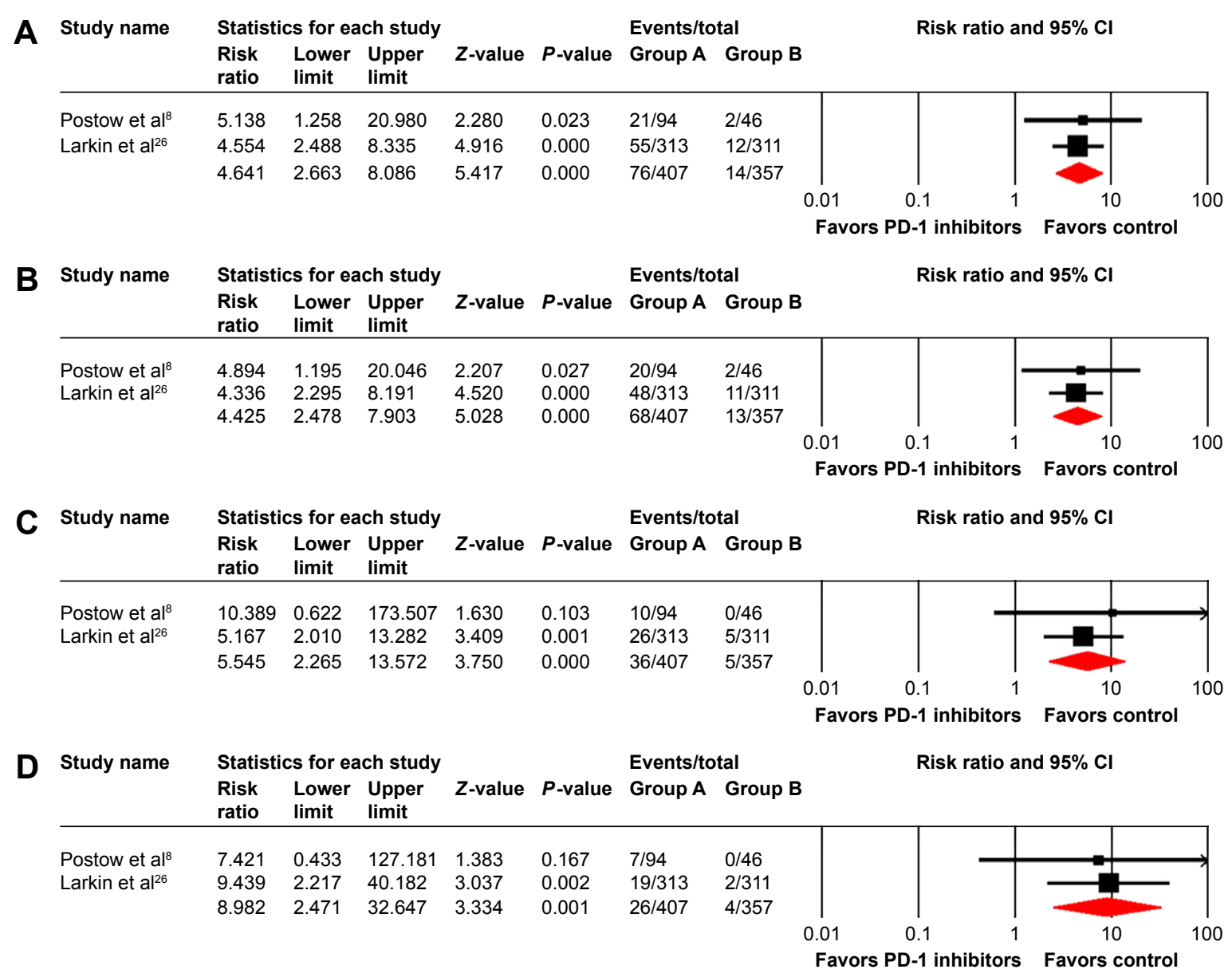

Figure 4 Relative risk of all-grade elevated ALT (A) and AST (B) and high-grade elevated ALT (C) and AST (D) for cancer patients receiving a nivolumab/ipilimumab combination compared with ipilimumab control.

Abbreviations: ALT, alanine aminotransferase; AST, aspartate aminotransferase; PD-I, program death I; Cl, confidence interval.

\section{RR of hepatic AEs in patients treated with nivolumab or pembrolizumab monotherapy versus ipilimumab control}

Patients treated with PD-1 inhibitors monotherapy (nivolumab or pembrolizumab) might have a lower risk of developing hepatic AEs compared with ipilimumab control. Then, we performed an analysis by nivolumab or pembrolizumab monotherapy versus ipilimumab. The RR of all-grade elevated ALT and AST was 0.91 (95\% CI: 0.52-1.59; $P=0.726$ ) (heterogeneity test: $P^{2}=0 \%, P=0.737$ ) and 1.27 (95\% CI: 0.70-2.30; $P=0.441$ ) (heterogeneity test: $P=0 \%$, $P=0.570$ ), respectively (Figure 5). Additionally, we observed an RR of 0.60 ( $95 \%$ CI: $0.19-1.88 ; P=0.38$ ) (heterogeneity test: $\left.I^{2}=0 \%, P=0.374\right)$ for high-grade elevated ALT and an RR of 0.77 (95\% CI: $0.18-3.20 ; P=0.716$ ) (heterogeneity test: $I^{2}=33.3 \%, P=0.221$ ) for high-grade elevated AST (Figure 5). Thus, no significant differences in the risk of all-grade or high-grade hepatic AEs can be detected between PD-1 inhibitors monotherapy and ipilimumab.

\section{Publication bias}

When stratified by PD-1 inhibitors monotherapy versus chemotherapy or everolimus control, no significant publication bias was detected for the RR of hepatic AEs by either Begg test or Egger test (RR of all-grade elevated ALT: Begg test $P=0.46$, Egger test $P=0.61 ; \mathrm{RR}$ of all-grade elevated AST: Begg test $P=0.46$, Egger test $P=0.62$; RR of highgrade elevated ALT: Begg test $P=0.26$, Egger test $P=0.42$; RR of high-grade elevated AST: Begg test $P=0.85$, Egger test $P=0.94$ ).

\section{Discussion}

Our meta-analysis of data from Phase II/III and III RCTs demonstrated that treatment with PD-1 inhibitors was associated with a significant increase in the risk of developing all-grade hepatic AEs, but not for high-grade hepatic AEs, which are different from the results previously reported by Abdel-Rahman et al. ${ }^{19}$ In their analysis, the use of immune checkpoint inhibitors (PD-1 inhibitors and CTLA-4 inhibitors) had a 


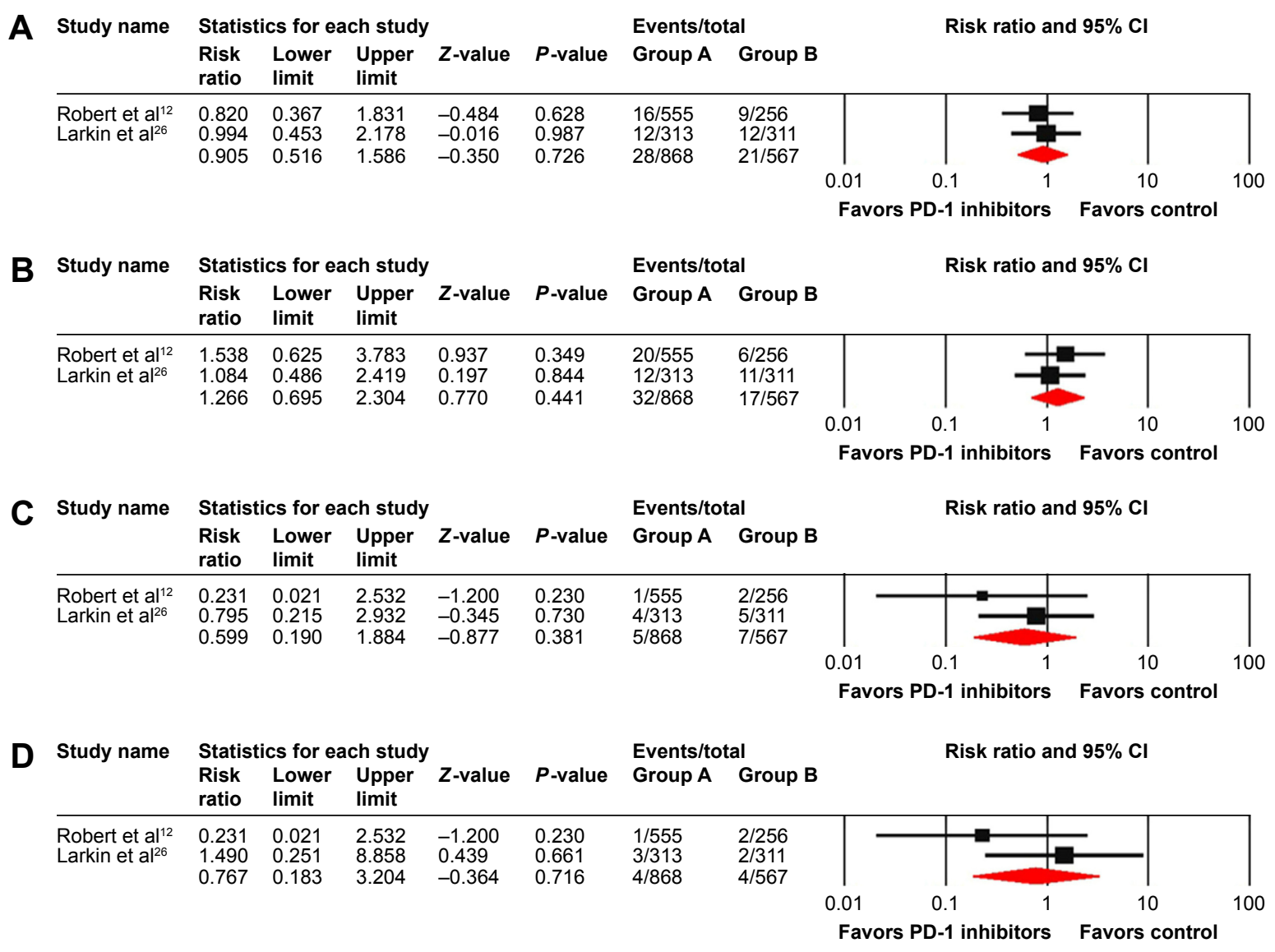

Figure 5 Relative risk of all-grade elevated ALT (A) and AST (B) and high-grade elevated ALT (C) and AST (D) for cancer patients receiving PD-I inhibitors monotherapy compared with ipilimumab control.

Abbreviations: ALT, alanine aminotransferase; AST, aspartate aminotransferase; PD-I, program death I; Cl, confidence interval.

causal relationship to an increased risk of high-grade elevated ALT and AST. The reason for this discrepancy could be partly explained by the distribution of treatment agents. All RCTs included in our study used PD-1 inhibitors as treatment drugs, while only three of ten RCTs in the study of Omar Abdel-Rahman et al evaluated PD-1 inhibitors (three RCTs evaluated nivolumab and seven RCTs evaluated CTLA-4 inhibitors). Although no significant differences in the risk of developing hepatic AEs can be detected between PD-1 inhibitors and ipilimumab in our analysis, these results should be interpreted cautiously as only two RCTs were included for the RR calculation. Moreover, it has been reported that anti-CTLA-4 monoclonal antibodies were associated with elevated AST and ALT levels in 10\% of patients or less, ${ }^{27-29}$ while this was $<5 \%$ for PD-1 inhibitors confirmed by our study. Additionally, we found that a nivolumab/ipilimumab combination was associated with significantly increased risk of developing all-grade and high-grade hepatic AEs compared with ipilimumab monotherapy. Similar to our results, Wolchok et $\mathrm{al}^{30}$ reported that high-grade treatmentrelated hepatic AEs were one of the most common AEs in patients who received ipilimumab plus nivolumab with $11 \%$ of patients for ALT and 13\% of patients for AST.

Hepatic AEs induced by PD-1/PD-L1 inhibitors commonly present as asymptomatic increase of AST and ALT, rarely total bilirubin. ${ }^{18}$ A proportion of patients may be presenting with fatigue, fever, and radiologic appearances including hepatomegaly, periportal lymphadenopathy, and periportal edema. ${ }^{31}$ Pathologic signs of pembrolizumab-induced hepatitis have been reported with periportal and lobular hepatitis, eosinophils, and some other inflammatory cell infiltrates. ${ }^{32}$ The median time to the onset of hepatic AEs was highly variable, such as 25 weeks (range 4-31 weeks) in lung cancer patients, 4 weeks (range $0.1-23$ weeks) in melanoma patients treated with nivolumab, and 19 weeks (range 0.3-93 weeks) in patients treated with pembrolizumab. ${ }^{33}$

The risk of hepatic AEs with anti-PD-1 therapy seems to be increased not only by combination use of anti-PD-1 and anti-CTLA-4 monoclonal antibody but also by combination use of PD-1 inhibitors and targeted therapy or chemotherapy. ${ }^{34}$ For example, nivolumab combined with either sunitinib or pazopanib was associated with increased 
incidence of high-grade AST/ALT elevations in metastatic renal cell carcinoma. ${ }^{35}$ In addition, higher rates of AST/ ALT elevation have been noted in advanced hepatocellular carcinoma patients treated with nivolumab. In a prospective analysis conducted by El-Khoueiry et al, ${ }^{36} 41$ patients were enrolled, $17 \%$ of patients experienced all-grade AST increase, and $15 \%$ of patients experienced all-grade ALT elevation. As for high-grade hepatic AEs, 12\% of patients suffered AST elevation and $10 \%$ of patients suffered ALT elevation.

Given hypothesized risk of triggering exacerbations of autoimmune diseases and/or chronic viral infections, patients with chronic hepatitis B/C (HBV/HCV), human immunodeficiency virus (HIV) infection, or autoimmune diseases have been excluded from clinical trials evaluating checkpoint blocking antibodies PD-1 and CTLA-4. Interestingly, Davar et $\mathrm{al}^{37}$ reported two patients with advanced melanoma and concomitant HCV or/and HIV infections treated with pembrolizumab and found that this drug was well tolerated with no exacerbation of underlying HCV/HIV infection or hepatic toxicity in both cases. Although the results of only two patients is in no way generalizable to other patients, it may indicate an important area for future investigation of the systematic evaluation of PD-1/PD-L1 inhibitors in patients with chronic $\mathrm{HBV} / \mathrm{HCV}$ and/or HIV infection and other entities including autoimmune disease requiring systemic immunosuppression.

Management of immune checkpoint inhibitor-related hepatic AEs consists of dose interruption and/or dose reduction and treatment with corticosteroids. Monitoring of patients for liver function before, during, and after antiPD-1 therapy is essential, but at the same time, the malignant disease in the liver, viral hepatitis, or another drug-induced toxicity should be excluded. Several algorithms have already been proposed for hepatic AEs with immune checkpoint blockade. ${ }^{28,33}$ The grade of the AE should be conducted to guide therapeutic decisions. In asymptomatic patients with AST or ALT $\leq 2.5 \times$ upper limit of normal, treatment with immunotherapy may continue without dose adjustment, but patients should be monitored closely for liver function. Immunotherapy should be held or reduced for grade 2, and oral prednisone may be involved in treatment. If a patient presents with grade 3 or 4 hepatic AEs, then discontinuation of immunotherapy should be considered in addition to methylprednisolone $2-4 \mathrm{mg} / \mathrm{kg} / \mathrm{d}$, and occasionally additional immune suppression with mycophenolate mofetil 500-1,000 mg every 12 hours is recommended. ${ }^{38}$ Infliximab, as confers its own risk of hepatotoxicity, should be avoided in this setting. ${ }^{1}$
However, there are several potential limitations needed to be considered in our meta-analysis. First, all the included studies were conducted at academic centers or major institutions, excluding patients with poor renal, hematological, and hepatic functions, so the true incidences and risk of PD-1 inhibitors-related hepatic AEs might be higher in daily clinical work. Second, this is a meta-analysis at study level and not on the patients' data, then confounding factors at the patient level including patients' comorbidities, previous therapeutic exposure, and concomitant treatments that affect liver function cannot be assessed properly and incorporated into the analysis. Third, this analysis is not powered to detect significant difference in the risk of developing hepatic AEs between PD-1 inhibitors monotherapy and ipilimumab control, while the observation might be influenced by sample size. Fourth, in this retrospective analysis, different tumor types, drug types, and dosage of PD-1 drug may increase the clinical heterogeneity among included trials and make the interpretation of the meta-analysis more problematic. However, the heterogeneity of pooled RR was not significant for both all-grade and high-grade hepatic AEs.

\section{Conclusion}

This meta-analysis of RCTs demonstrates that the use of PD-1 inhibitors is associated with an increased risk of hepatic AEs in cancer patients, which is primarily for lower grade events. And the risk of hepatic AEs is higher in patients treated with a nivolumab/ipilimumab combination compared with ipilimumab control. These findings would help clinicians and patients to recognize the risk of hepatic AEs of PD-1 checkpoint inhibitors to avoid delays in diagnosis and treatment.

\section{Disclosure}

The authors report no conflicts of interest in this work.

\section{References}

1. Postow MA, Callahan MK, Wolchok JD. Immune checkpoint blockade in cancer therapy. J Clin Oncol. 2015;33(17):1974-1982.

2. Taube JM, Klein A, Brahmer JR, et al. Association of PD-1, PD-1 ligands, and other features of the tumor immune microenvironment with response to anti-PD-1 therapy. Clin Cancer Res. 2014;20(19): 5064-5074.

3. Zou W, Chen L. Inhibitory B7-family molecules in the tumour microenvironment. Nat Rev Immunol. 2008;8(6):467-477.

4. Ansell SM, Lesokhin AM, Borrello I, et al. PD-1 blockade with nivolumab in relapsed or refractory Hodgkin's lymphoma. $N$ Engl J Med. 2015; 372(4):311-319.

5. Herbst RS, Baas P, Kim DW, et al. Pembrolizumab versus docetaxel for previously treated, PD-L1-positive, advanced non-small-cell lung cancer (KEYNOTE-010): a randomised controlled trial. Lancet. 2016; 387(10027):1540-1550. 
6. Hamanishi J, Mandai M, Ikeda T, et al. Efficacy and safety of anti-PD-1 antibody (nivolumab: BMS-936558, ONO-4538) in patients with platinum-resistant ovarian cancer. J Clin Oncol. 2014;32(15 suppl):5511.

7. Weber JS, D'Angelo SP, Minor D, et al. Nivolumab versus chemotherapy in patients with advanced melanoma who progressed after anti-CTLA-4 treatment (CheckMate 037): a randomised, controlled, open-label, phase 3 trial. Lancet Oncol. 2015;16(4):375-384.

8. Postow MA, Chesney J, Pavlick AC, et al. Nivolumab and ipilimumab versus ipilimumab in untreated melanoma. $N$ Engl J Med. 2015; 372(21):2006-2017.

9. Borghaei H, Paz-Ares L, Horn L, et al. Nivolumab versus docetaxel in advanced nonsquamous non-small-cell lung cancer. $N$ Engl J Med. 2015;373(17):1627-1639.

10. Brahmer J, Reckamp KL, Baas P, et al. Nivolumab versus docetaxel in advanced squamous-cell non-small-cell lung cancer. $N$ Engl J Med. 2015;373(2):123-135.

11. Motzer RJ, Escudier B, McDermott DF, et al; CheckMate 025 Investigators. Nivolumab versus everolimus in advanced renal-cell carcinoma N Engl J Med. 2015;373(19):1803-1813.

12. Robert C, Schachter J, Long GV, et al; KEYNOTE-006 Investigators. Pembrolizumab versus ipilimumab in advanced melanoma. $N$ Engl J Med. 2015;372(26):2521-2532.

13. Garon EB, Rizvi NA, Hui R, et al; KEYNOTE-001 Investigators. Pembrolizumab for the treatment of non-small-cell lung cancer. $N$ Engl J Med. 2015;372(21):2018-2028.

14. McDermott DF, Sosman JA, Sznol M, et al. Atezolizumab, an antiprogrammed death-ligand 1 antibody, in metastatic renal cell carcinoma: long-term safety, clinical activity, and immune correlates from a phase Ia study. J Clin Oncol. 2016;34(8):833-842.

15. Fehrenbacher L, Spira A, Ballinger M, et al; POPLAR Study Group Atezolizumab versus docetaxel for patients with previously treated non-small-cell lung cancer (POPLAR): a multicentre, open-label, phase 2 randomised controlled trial. Lancet. 2016;387(10030):1837-1846.

16. Rizvi N, Brahmer J, Ou S-HI. Safety and clinical activity of MEDI4736, an anti-programmed cell death-ligand 1 (PD-L1) antibody, in patients with non-small cell lung cancer (NSCLC). Proc Am Soc Clin Oncol. 2015;33(15 suppl):abstr8032.

17. Brahmer JR, Tykodi SS, Chow LQ, et al. Safety and activity of antiPD-L1 antibody in patients with advanced cancer. N Engl J Med. 2012; 366(26):2455-2465.

18. Postow MA. Managing immune checkpoint-blocking antibody side effects. Am Soc Clin Oncol Educ Book. 2015:76-83.

19. Abdel-Rahman O, ElHalawani H, Fouad M. Risk of elevated transaminases in cancer patients treated with immune checkpoint inhibitors: a meta-analysis. Expert Opin Drug Saf. 2015;14(10):1507-1518.

20. Moher D, Liberati A, Tetzlaff J, Altman DG; PRISMA Group. Preferred reporting items for systematic reviews and meta-analyses: the PRISMA statement. Int J Surg. 2010;8(5):336-341.

21. Jadad AR, Moore RA, Carroll D, et al. Assessing the quality of reports of randomized clinical trials: is blinding necessary? Control Clin Trials. 1996;17(1):1-12.
22. DerSimonian R, Laird N. Meta-analysis in clinical trials. Control Clin Trials. 1986;7(3):177-188.

23. Lau J, Ioannidis JP, Schmid CH. Quantitative synthesis in systematic reviews. Ann Intern Med. 1997;127(9):820-826.

24. Higgins JP, Thompson SG, Deeks JJ, Altman DG. Measuring inconsistency in meta-analyses. BMJ. 2003;327(7414):557-560.

25. Robert C, Long GV, Brady B, et al. Nivolumab in previously untreated melanoma without BRAF mutation. $N$ Engl J Med. 2015;372(4): 320-330.

26. Larkin J, Chiarion-Sileni V, Gonzalez R, et al. Combined nivolumab and ipilimumab or monotherapy in untreated melanoma. $N$ Engl J Med. 2015;373(1):23-34.

27. Ribas A, Kefford R, Marshall MA, et al. Phase III randomized clinical trial comparing tremelimumab with standard-of-care chemotherapy in patients with advanced melanoma. J Clin Oncol. 2013;31(5): 616-622.

28. Hodi FS, O’Day SJ, McDermott DF, et al. Improved survival with ipilimumab in patients with metastatic melanoma. $N$ Engl J Med. 2010; 363(8):711-723.

29. Wolchok JD, Neyns B, Linette G, et al. Ipilimumab monotherapy in patients with pretreated advanced melanoma: a randomised, double-blind, multicentre, phase 2, dose-ranging study. Lancet Oncol. 2010;11(2): $155-164$.

30. Wolchok JD, Kluger H, Callahan MK, et al. Nivolumab plus ipilimumab in advanced melanoma. N Engl J Med. 2013;369(2):122-133.

31. Kim KW, Ramaiya NH, Krajewski KM, et al. Ipilimumab associated hepatitis: imaging and clinicopathologic findings. Invest New Drugs. 2013;31(4):1071-1077.

32. Hofmann L, Forschner A, Loquai C, et al. Cutaneous, gastrointestinal, hepatic, endocrine, and renal side-effects of anti-PD-1 therapy. Eur $J$ Cancer. 2016;60:190-209.

33. Eigentler TK, Hassel JC, Berking C, et al. Diagnosis, monitoring and management of immune-related adverse drug reactions of anti-PD-1 antibody therapy. Cancer Treat Rev. 2016;45:7-18.

34. Bendell JC, Powderly JD, Lieu CH, et al. Safety and efficacy of MPDL3280A (antiPDL1)in combination with bevacizumab (bev) and/or FOLFOX in patients (pts) with metastatic colorectal cancer (mCRC). J Clin Oncol. 2015;33:15s.

35. Amin A, Plimack ER, Infante JR, et al. Nivolumab (anti-PD-1; BMS936558, ONO-4538) in combination with sunitinib or pazopanib in patients (pts) with metastatic renal cell carcinoma (mRCC). J Clin Oncol. 2014;32:5s.

36. El-Khoueiry AB, Melero I, Crocenzi TS, et al. Phase I/II safety and antitumor activity of nivolumab in patients with advanced hepatocellular carcinoma (HCC): CA209-040. J Clin Oncol. 2015;33:15s.

37. Davar D, Wilson M, Pruckner C, Kirkwood JM. PD-1 blockade in advanced melanoma in patients with hepatitis C and/or HIV. Case Rep Oncol Med. 2015;2015:737389.

38. Naidoo J, Page DB, Li BT, et al. Toxicities of the anti-PD-1 and anti-PD-L1 immune checkpoint antibodies. Ann Oncol. 2015;26(12): $2375-2391$.

\section{Publish your work in this journal}

Drug Design, Development and Therapy is an international, peerreviewed open-access journal that spans the spectrum of drug design and development through to clinical applications. Clinical outcomes, patient safety, and programs for the development and effective, safe, and sustained use of medicines are the features of the journal, which

\section{Dovepress}

has also been accepted for indexing on PubMed Central. The manuscript management system is completely online and includes a very quick and fair peer-review system, which is all easy to use. Visit http://www.dovepress.com/testimonials.php to read real quotes from published authors. 